\title{
Theory of neutrino detection: flavor oscillations and weak values
}

\author{
Yago P. Porto-Silva ${ }^{1,2, a}$, M. C. de Oliveira ${ }^{1, b}{ }_{(\mathbb{C}}$ \\ ${ }^{1}$ Instituto de Física "Gleb Wataghin", Universidade Estadual de Campinas, Campinas, SP, Brazil \\ ${ }^{2}$ Max-Planck-Institut für Kernphysik, 69117 Heidelberg, Germany
}

Received: 12 October 2020 / Accepted: 31 March 2021 / Published online: 19 April 2021

(c) The Author(s) 2021

\begin{abstract}
We revisit the theory of neutrino oscillations and describe it through the formalism of weak measurements with postselection. It is well understood that due to the large momentum uncertainty in detection, there is no collapse of the neutrino wavefunction in the momentum or energy basis, and the mass eigenstates are detected coherently. Here we show that postselection, which projects the system to a final flavor state, deforms the system wavefunction in such a way that the momentum detected is not the expectation value of the neutrino mass eigenstates momenta, but the corresponding weak value. We use the weak values to describe the intermediate state in the oscillation process, avoiding problems in defining probability currents for particle states with mass superposition.
\end{abstract}

\section{Introduction}

Neutrino oscillation is the phenomenon by which neutrinos undergo periodic flavor transformations as they propagate [1-3]. Its simplest description is a model in which the flavor states are not eigenstates of the propagation Hamiltonian, and, as a consequence, the flavor content changes with time and distance [4-6]. Most of the literature treats the eigenstates of propagation as plane waves: states with definite mass, energy, and momentum. Although extremely useful, the plane wave description neglects an essential ingredient in modelling the neutrino detection, which is the energy uncertainty.Many authors have incorporated the energy uncertainty using quantum mechanical (QM) wave-packets [7-18] and quantum field theory [19-27]. For the measurement theory in this paper, we shall use Gaussian wave-packets.

The uncertainties in energy and momentum of a massive neutrino wave-packet come from the approximate conservation of mean energies and momenta of all particles in the

\footnotetext{
a e-mail: yporto@ifi.unicamp.br

b e-mail: marcos@ifi.unicamp.br (corresponding author)
}

production and detection processes. When these uncertainties are large enough so that one cannot, even in principle, resolve the neutrino masses, the produced and detected neutrino states can be written as a coherent superposition of mass eigenstates. When neutrinos are detected, their flavor is revealed by the charged leptons produced in the interaction, and their energy and momentum can, in principle, be reconstructed by measuring energies and momenta of all other particles involved in the detection process. Even if all this information is inaccessible to the experimentalist, it is available to particles in the detection process, and this, by itself, configures a measurement. However, from QM, two incompatible observables (i.e. non-commuting observables) are being measured at the same time in this detection process: flavor and energy (and momentum). ${ }^{1}$ The most critical consequence of measuring, or inferring two incompatible observables at the same time is that they randomly mess up information about each other, being manifestly complementary. Therefore, the measurement of energy-momentum of neutrinos should disturb previous information about flavor, particularly the flavor transitions during the propagation. Disturbance is prevented, however, due to the large uncertainties in energy and momentum in the detection process. Indeed, this type of measurement, with large uncertainties, is called weak measurement [28-30], and its main feature is to disturb very little the quantum state of the system, not degrading the information about complementary observables. ${ }^{2}$

Based on weak measurements plus postselection [31-33], i.e., the fact that usually only one flavor type is measured in the end, we revisit the theory of neutrino detection and notice that flavor neutrinos obey probability currents with momentum and energy described by weak values. ${ }^{3}$ To find

\footnotetext{
${ }^{1}$ In the following sections, we treat neutrino propagation in one dimension where there is one to one relation between energy and momentum.

2 The concept of weak measurements has nothing to do with the concept of weak interactions in the Standard Model.

3 Weak values are the result of weak measurements with postselection. They were previously explored inneutrino oscillations when
} 
such currents was regarded as a conceptual issue in previous references once in quantum mechanics, Hilbert spaces and probability currents are defined only for particles of definite mass, that is not the case of neutrinos. References [34-37] present possibilities to get around the issue, and here we show that as soon as we interpret the process as a weak measurement with postselection we do not meet such limitations. It is indeed interesting to see how postselection theories "violate" a number of principles of standard quantum mechanics [38].

This paper develops as follows. Firstly, we review the standard procedures for describing neutrino wave-packets emission and detection and the resulting observed neutrino oscillations; then, we review the von Neumann measurements and the weak measurement regime. With the fundamentals established, we develop the interpretation of neutrino oscillations under the weak measurement regime, demonstrating how the neutrino probability current is described; and to finish, we present our conclusions and perspectives.

\section{Neutrino wave-packets}

In this section, we review the standard wave-packet formalism of neutrino oscillations in one dimension. ${ }^{4}$ We use natural units $(\hbar=c=1)$ throughout the paper.

Consider a process at (average) coordinates $(t=0, x=$ $0)$ that produces a neutrino of flavor $\alpha$ which propagates and is detected at $(T, L)$ with flavor $\beta$. Using a normalized Gaussian envelope, we can write the one-particle state of the neutrino produced at the origin:

$$
\begin{aligned}
\left|v_{\alpha}^{P}\right\rangle & =\sum_{a} U_{\alpha a}^{*}\left|v_{a}^{P}\right\rangle \\
& =\sum_{a} U_{\alpha a}^{*} \int \frac{d p}{\sqrt{4 \pi E_{a}(p)}} \phi^{P}\left(p-p_{a}\right)\left|v_{a}(p)\right\rangle,
\end{aligned}
$$

with $E_{a}(p)=\sqrt{p^{2}+m_{a}^{2}}$ and

$\phi^{P}\left(p-p_{a}\right)=\frac{1}{\left(2 \pi \sigma_{p P}^{2}\right)^{\frac{1}{4}}} e^{-\frac{\left(p-p_{a}\right)^{2}}{4 \sigma_{p P}^{2}}}$,

such that $\int d p\left|\phi^{P}\left(p-p_{a}\right)\right|^{2}=1$. Here the flavor eigenstate $\left|v_{\alpha}^{P}\right\rangle$ is a superposition of mass eigenstate wave-packets, $\left|v_{a}^{P}\right\rangle$, with mass $m_{a}$, weightened by the complex-conjugated PMNS matrix elements, $U_{\alpha a}^{*}$. The mass eigenstates themselves are a superposition of energy and momentum eigen-

footnote 3 continued

superluminal velocity was reported [49] and references therein. Here we develop it from a foundational point of view, highlighting concepts of quantum measurement theory.

4 This is a good approximation for cases in which the distance between neutrino source and detector is large compared to their size [26]. states $\left|v_{a}(p)\right\rangle$. In the $x$-space, $v_{a}$ wave function, at time $t$, is given by [39]:

$\left\langle x \mid v_{a}^{P}(t)\right\rangle=\int \frac{d p}{\sqrt{4 \pi E_{a}(p)}} \phi^{P}\left(p-p_{a}\right) e^{-i E_{a}(p) t} e^{i p x}$.

The average momenta and momentum uncertainties of different mass eigenstates, $p_{a}$ and $\sigma_{p P}$, respectively, are determined by the kinematics and the properties of the particles involved in the production $(P)$ process. We assume that all mass eigenstates are extremely relativistic, $p_{a}>>m_{a}$, so that we can approximate their average energies by [39],

$\epsilon_{a} \approx E+\xi \frac{m_{a}^{2}}{2 E}$

in which $E$ is the energy determined by the kinematics of the production process if neutrino masses are neglected and

$\frac{\xi}{2 E}=\left.\frac{\partial \epsilon_{a}}{\partial m_{a}^{2}}\right|_{m_{a}=0}$

is the coefficient of the first-order term if one expands $\epsilon_{a}=$ $\sqrt{p_{a}^{2}+m_{a}^{2}}$ around $m_{a}=0$. The corresponding momenta are

$p_{a} \approx E-(1-\xi) \frac{m_{a}^{2}}{2 E}$

For a given process, $\xi$ can be calculated from energymomentum conservation up to order $\frac{m_{a}^{2}}{E^{2}}$.

The effective momentum-space uncertainty of the produced neutrino wave-packets $\sigma_{p P}$ is

$\sigma_{p P} \sim \min \left\{\delta_{p P}, \delta_{e P}\right\}$

where $\delta_{p P}$ and $\delta_{e P}$ are, respectively, the momentum and energy uncertainties in the production process. In configuration space, $\sigma_{x P}=\frac{1}{2 \sigma_{p P}}$.

The detection process, in the standard formalism, is considered by propagating the ket in (1) from the origin to $(T, L)$ and then projecting it on the state

$$
\left|v_{\beta}^{D}\right\rangle=\sum_{a} U_{\beta a}^{*} \int \frac{d p}{\sqrt{2 \pi} \sqrt{2 E_{a}(p)}} \phi^{D}\left(p-p_{a}\right)\left|v_{a}(p)\right\rangle,
$$

with

$\phi^{D}\left(p-p_{a}\right)=\frac{1}{\left(2 \pi \sigma_{p D}^{2}\right)^{\frac{1}{4}}} e^{-\frac{\left(p-p_{a}\right)^{2}}{4 \sigma_{p D}^{2}}}$, 
which takes into account the effective momentum-space uncertainty $\sigma_{p D}$ of the detection $(D)$ wave-packet, related to $\delta_{p D}$ and $\delta_{e D}$ in a similar way to (7),

$\sigma_{p D} \sim \min \left\{\delta_{p D}, \delta_{e D}\right\}$

and $\sigma_{x D}=\frac{1}{2 \sigma_{p D}}$. Notice that $\int d p\left|\phi^{D}\left(p-p_{a}\right)\right|^{2}=1$. The average momentum $p_{a}$ seen in the detection process is determined by the kinematics of the production process. ${ }^{5}$ Notice that (1) and (8) are normalized independently.

Now, we compute

$$
\begin{aligned}
A_{\alpha \beta}(L, T) & =\left\langle v_{\beta}^{D} e^{-i \mathbf{H} T+i \mathbf{p} L} \mid v_{\alpha}^{P}(0,0)\right\rangle \\
& =\left\langle v_{\beta}^{D} \mid v_{\alpha}^{P}(L, T)\right\rangle,
\end{aligned}
$$

i.e., the amplitude of probability of detection of neutrinos in state $\left|v_{\beta}^{D}\right\rangle$ when they were generated in state $\left|v_{\alpha}^{P}(0,0)\right\rangle$ after traveling the distance $L$ during the time interval $T$, being $\mathbf{H}$ the Hamiltonian and $\mathbf{p}$ the momentum operators. Using the condition $\left\langle v_{a}(p) \mid v_{a}\left(p^{\prime}\right)\right\rangle=(2 \pi) 2 E_{a}(p) \delta\left(p-p^{\prime}\right)$ :

$A_{\alpha \beta}(L, T)=\sum_{a} \frac{U_{\alpha a}^{*} U_{\beta a}}{\sqrt{2 \pi \sigma_{p P} \sigma_{p D}}} \int d p e^{-\frac{\left(p-p_{a}\right)^{2}}{4 \sigma_{p}^{2}}} e^{-i E_{a}(p) T+i p L}$.

Let us consider sharply peaked Gaussian functions in momentum space, with average momentum and energy given by (4) and (6). ${ }^{6}$ In this context, the relativistic dispersion relation can be approximated by $E_{a}(p) \approx \epsilon_{a}+v_{a}\left(p-p_{a}\right)$, with

$v_{a}=\left.\frac{\partial E_{a}(p)}{\partial p}\right|_{p=p_{a}}=\frac{p_{a}}{\epsilon_{a}} \approx 1-\frac{m_{a}^{2}}{2 E^{2}}$.

Thus,

$$
\begin{aligned}
A_{\alpha \beta}(L, T)= & \sqrt{\frac{2 \sigma_{x P} \sigma_{x D}}{\sigma_{x}^{2}}} \sum_{a} U_{\alpha a}^{*} U_{\beta a} \\
& \times \exp \left(-i \epsilon_{a} T+i p_{a} L-\frac{\left(L-v_{a} T\right)^{2}}{4 \sigma_{x}^{2}}\right) .
\end{aligned}
$$

In (14), $\sigma_{x}$ is the effective size of the detection region that takes into account space and time intervals in which the neutrino and all particles in the detection process are overlapped. Since the neutrino that reached the detection process carries information about the production process, $\sigma_{x}$ takes into account features of both production and detection, in a similar

\footnotetext{
5 This constrain can be relaxed, see [26]

${ }^{6}$ Dispersion due to different phase velocities is negligible [25].
}

way to $\sigma_{p}$ - the effective resolution with which the detection process can measure momentum: ${ }^{7}$

$\sigma_{x}^{2}=\sigma_{x P}^{2}+\sigma_{x D}^{2}$ and $\quad \frac{1}{\sigma_{p}^{2}}=\frac{1}{\sigma_{p P}^{2}}+\frac{1}{\sigma_{p D}^{2}}$

Both are related by $\sigma_{x} \sigma_{p}=\frac{1}{2}$.

Squaring the amplitude in (14) and integrating out the $T$ dependence, we obtain

$$
\begin{aligned}
& P_{\alpha \beta}(L)=\frac{2 \sigma_{x} P \sigma_{x} D}{\sigma_{x}^{2}} \sum_{a, b} U_{\alpha a}^{*} U_{\beta a} U_{\alpha b} U_{\beta b}^{*} e^{i(1-\xi) \frac{\Delta m_{a b}^{2}}{2 E} L} \\
& \times \int d T \exp \left[-\frac{\left(L-v_{a} T\right)^{2}+\left(L-v_{b} T\right)^{2}}{4 \sigma_{x}^{2}}\right] e^{-i \xi \frac{\Delta m_{a b}^{2}}{2 E} T} .
\end{aligned}
$$

After integration, we substitute the expression in (13) for the velocity of relativistic mass eigenstates in the exponents, preserving the terms of first order in $\frac{m_{a}^{2}}{E^{2}}$ (or one order higher if the first order vanishes) and find

$$
\begin{aligned}
P_{\alpha \beta}(L)= & \frac{2 \sqrt{2 \pi} \sigma_{x P} \sigma_{x D}}{\sigma_{x}} \\
& \times \sum_{a, b} \sqrt{\frac{2}{v_{a}^{2}+v_{b}^{2}}} U_{\alpha a}^{*} U_{\beta a} U_{\alpha b} U_{\beta b}^{*} \\
& \times e^{-i \frac{\Delta m_{a b}^{2} L}{2 E}} e^{-\left(\frac{L}{L_{c o h}^{a b}}\right)^{2}} e^{-\frac{\left(\Delta \epsilon_{a b}\right)^{2}}{8 \sigma_{e}^{2}}},
\end{aligned}
$$

with

$\Delta \epsilon_{a b}=\xi \frac{\Delta m_{a b}^{2} L}{2 E}, \quad$ and $\quad L_{c o h}^{a b}=\frac{4 \sqrt{2} E^{2}}{\left|\Delta m_{a b}^{2}\right|} \sigma_{x}$

where $\sigma_{e}^{2} \approx \frac{1}{2}\left(v_{a}^{2}+v_{b}^{2}\right) \sigma_{p}^{2}$.

The probability in (17) is not normalized [18,26], and its magnitude is dependent on the sizes of the produced and detected wave-packets and their overlap. Indeed,

$$
\begin{aligned}
\sum_{\beta} P_{\alpha \beta}(L) & =\frac{2 \sqrt{2 \pi} \sigma_{x P} \sigma_{x D}}{\sigma_{x}} \sum_{a} \frac{\left|U_{\alpha a}\right|^{2}}{v_{a}} \\
& \approx \frac{2 \sqrt{2 \pi} \sigma_{x P} \sigma_{x D}}{\sigma_{x}} .
\end{aligned}
$$

In addition, it is not dimensionless but has a unit of length. As we will see in next sections, this is not a problem if we interpret the mass neutrino wavefunctions as pointer states in the von Neumann sense, unitarity will be automatically obeyed. $^{8}$

\footnotetext{
${ }^{7}$ In the literature $\sigma_{x}$ is most commonly referred as the size of the wavepacket. Here we want to emphasize that it is related to the momentum resolution in the detection process.

8 A number of references overcome this problem by using quantum field theory, for instance check $[18,26]$.
} 


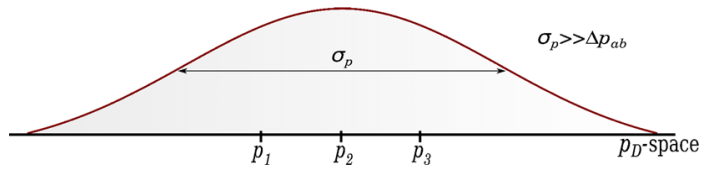

Fig. 1 Illustration of the condition for coherent production and detection of neutrinos, $\Delta p_{a b}<<\sigma_{p}$, with $p_{1}, p_{2}$ and $p_{3}$ the mean momenta of the massive neutrino wave-packets given in (6)

The discussion about the physical meaning of the exponentials (17) can be found in many references $[11,13,18,25$, 39]. Here we highlight:

- The exponential $e^{-\left(\frac{L}{L_{c o h}^{a b}}\right)^{2}}$ defines the coherence length, $L_{c o h}^{a b}$, that is the effective distance after which mass eigenstates $v_{a}$ and $v_{b}$ lose coherence due to separation of their wave-packets. For $L<<L_{c o h}^{a b}$ wave-packet separation is negligible.

- The term $e^{-\frac{\left(\Delta \epsilon_{a b}\right)^{2}}{8 \sigma_{e}^{2}}}$ defines the conditions under which neutrinos are produced and detected coherently. In the limit,

$$
\Delta \epsilon_{a b}<<\sigma_{e}, \quad \text { (Coherence Condition) }
$$

the conditions for coherent production and detection of the mass eigenstates $v_{a}$ and $v_{b}$ are set (see Fig. 1). In the relativistic regime and in one dimension, (20) is equivalent to $\Delta p_{a b}<<\sigma_{p}[17]$.

Therefore, $L<<L_{c o h}^{a b}$ and $\Delta_{a b}<<\sigma_{e}$ are usually referred as the conditions for the observability of neutrino oscillations.

\section{Weak measurements and weak values}

In this section, we formalize the concept of quantum measurement in the von Neumann regime and use it to distinguish between the strong (great disturbance, wavefunction collapse) and weak measurements (very little disturbance) [28-33].

\section{1 von Neumann measurements}

In the von Neumann measurement model $[40,41]$, the measuring device (or pointer) is a secondary quantum system with canonical variables $\mathbf{x}_{D}$ and $\mathbf{p}_{D}$ satisfying $\left[\mathbf{x}_{D}, \mathbf{p}_{D}\right]=i$, in natural units. The Hamiltonian that describes the interaction between the system and the device usually couples the observable of interest, that we call $\mathbf{A}$,with some of the canonical variables, $\mathbf{x}_{D}$, for example, so that the change in the conjugate variable, $\mathbf{p}_{D}$, reveals information about $\mathbf{A}$. The interaction Hamiltonian can be written as

$\mathbf{H}_{i n t}=-\delta\left(t-t_{0}\right) \mathbf{A} \mathbf{x}_{D}$,

where the delta function assures the interaction to happen for times only in the vicinity of $t_{0}$ while, at any other instant, the system evolves freely. To illustrate how the pointer variable, $\mathbf{p}_{D}$ in our example, acquires information about $\mathbf{A}$, we compute its evolution at times close to $t_{0}$ in the Heisenberg picture:

$$
\begin{aligned}
\frac{d}{d t} \mathbf{p}_{D}(t) & =i\left[\mathbf{H}_{i n t}, \mathbf{p}_{D}(t)\right] \\
& =-i \delta\left(t-t_{0}\right) \mathbf{A}(t)\left[\mathbf{x}_{D}(t), \mathbf{p}_{D}(t)\right] \\
& =\delta\left(t-t_{0}\right) \mathbf{A}(t),
\end{aligned}
$$

then,

$\mathbf{p}_{D}\left(t>t_{0}\right)-\mathbf{p}_{D}\left(t<t_{0}\right)=\int d t \delta\left(t-t_{0}\right) \mathbf{A}(t)=\mathbf{A}\left(t_{0}\right)$.

Therefore, the change in the pointer immediately after $t_{0}$ gives the information about the status of the observer of interest at $t_{0}$.

\subsection{Statistics of the pointer variable}

Consider an ensemble defined by a system prepared in state $\left|\psi_{i}\right\rangle$ and measuring device in state $|\phi\rangle$. A system ensemble prepared in a specific initial state defines a preselected ensemble. We know the effect of the measurement on the device is to change the status of its pointer variable $\mathbf{p}_{D}$ proportionally to the system observable of interest $\mathbf{A}$ according to (23). Starting from the initial state of the system plus measuring device, $\left|\Omega_{i}\right\rangle=\left|\psi_{i}\right\rangle|\phi\rangle$, we find that the impact of the measurement on this state is given by

$\left|\Omega_{f}\right\rangle=e^{-i \int \mathbf{H}_{i n t} d t}\left|\Omega_{i}\right\rangle=e^{i \mathbf{A x}_{D}}\left|\psi_{i}\right\rangle|\phi\rangle$.

Projecting (24) into the pointer variable space

$$
\begin{aligned}
\left\langle p_{D} \mid \Omega_{f}\right\rangle & =\sum_{a}|a\rangle\left\langle a \mid \psi_{i}\right\rangle\left\langle p_{D} e^{i a \mathbf{x}_{D}} \mid \phi\right\rangle \\
& =\sum_{a}|a\rangle\left\langle a \mid \psi_{i}\right\rangle \phi\left(p_{D}-a\right),
\end{aligned}
$$

in which $\{|a\rangle\}$ are the eigenvectors of operator $\mathbf{A}$, and $\phi\left(p_{D}-a\right)=\left\langle p_{D}-a \mid \phi\right\rangle$ is the shifted (by $\left.a\right)$ wavefunction, $\phi\left(p_{D}\right)$, due to the action of the translation operator $e^{i a \mathbf{x}_{D}}$. The probability distribution of the pointer apparatus state after the measurement is given by the absolute square of (25) 


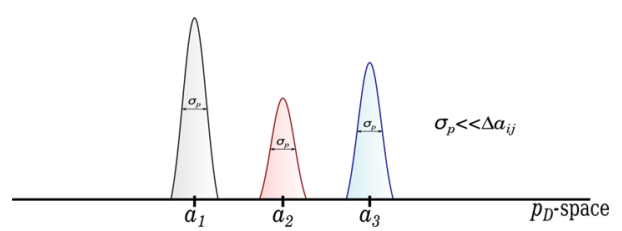

Fig. 2 Illustration of a pointer wavefunction that can resolve the spectrum of eigenvalues of the system observable $\mathbf{A}$ in a strong measurement, $\sigma_{p}<<\Delta a_{i j}$. The amplitude of the distributions are proportional to the probability amplitude of the system to be in the state $\left|a_{i}\right\rangle$. In the limiting case of $\sigma_{p} \rightarrow 0$, one recovers Born's rule. Detector and system are fully entangled immediately after the measurement

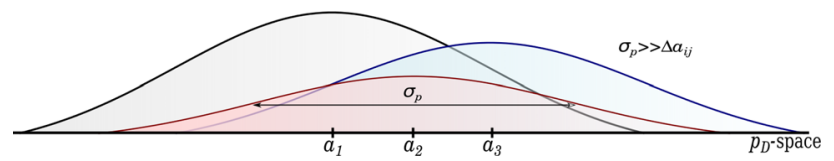

Fig. 3 Illustration of a pointer wavefunction that cannot resolve the spectrum of eigenvalues of the system observable $\mathbf{A}$ in a weak measurement, $\sigma_{p}>\Delta a_{i j}$. The amplitude of the distributions are proportional to the probability amplitude of the system to be in the state $\left|a_{i}\right\rangle$. Because of the poor resolution, detector and system are not fully entangled and the system wavefunction is very little disturbed after the measurement

$$
P_{f}\left(p_{D}\right)=\left|\left\langle p_{D} \mid \Omega_{f}\right\rangle\right|^{2}=\sum_{a}\left|\left\langle a \mid \psi_{i}\right\rangle\right|^{2}\left|\phi\left(p_{D}-a\right)\right|^{2} .
$$

Remark that for the probability interpretation to hold, we need a normalized pointer wavefunction,

$\langle\phi \mid \phi\rangle=\int d p_{D}\left|\phi\left(p_{D}\right)\right|^{2}=1$.

What we call strong or weak measurement depends very much on the spread of the apparatus wavefunction in the $p_{D^{-}}$ space, $\sigma_{p}$, relative to the separation, $\Delta a_{i j}$, of the eigenvalues, $\left\{a_{i}\right\}$, of the system. ${ }^{9}$ If the pointer can resolve the spectrum, in other words, if

$\sigma_{p}<<\Delta a_{i j}, \quad$ (Strong measurement)

it is called strong measurement, and is pictorially represented in Fig. 2.

In the opposite limit,

$\sigma_{p}>>\Delta a_{i j} \quad$ (Weak measurement),

we say that the system is weakly measured by the apparatus, see Fig. 3.

Weak measurements were first proposed as a way in which one can extract average state information without entirely collapsing the system [41]. In fact, due to large uncertainty $\sigma_{p}$ in the apparatus wavefunction, after measurement, the state of the apparatus is not strongly correlated or entangled

\footnotetext{
${ }^{9}$ It is common to model the pointer with a Gaussian wavefunction.
}

with any of the states $\left\{\left|a_{i}\right\rangle\right\}$ of the system. This can be seen graphically in Fig 3. For comparison, observe how the states of the system and apparatus are fully correlated after a strong measurement (resembling Born's rule) in Fig. 2.

A useful way of thinking about weak measurements is that the eigenvalues of $\mathbf{A}$ are so close that the effect of the translation operator on the pointer is very small. More precisely, suppose our pointer just measured $a_{1}$ (went from zero to $a_{1}$ by means of $e^{i a_{1} \mathbf{x}_{D}}$ ) in Fig. 3, to move its center to $a_{2}$ we operate with:

$e^{i\left(a_{2}-a_{1}\right) \mathbf{x}_{D}}=1+i \Delta a_{21} \mathbf{x}_{D}-\frac{1}{2}\left(\Delta a_{21}\right)^{2} \mathbf{x}_{D}^{2}+\cdots$

with $\Delta a_{21}=a_{2}-a_{1}$. However, due to (29),

$\left\langle\phi\left(\Delta a_{21}\right)^{2} \mathbf{x}_{D}^{2} \mid \phi\right\rangle=\left(\Delta a_{21}\right)^{2} \sigma_{x}^{2} \approx \frac{\left(\Delta a_{21}\right)^{2}}{\sigma_{p}^{2}}<<1$.

Hence, in case the measurement is weak, it is enough to use the expansion in (30) up to first order.

Although strong and weak measurements are conceptually different, they are quantitatively equivalent with respect to expectation values [42]. In other words,

$\left\langle\Omega_{f}\left|\mathbf{p}_{D}\right| \Omega_{f}\right\rangle=\langle\mathbf{A}\rangle \psi_{i}$,

independently of $\sigma_{p}$.

\subsection{Pre- and postselected ensembles}

If the expectation values of the observables of the system are the same independently of the measurement type (see (32)), then one can judge unnecessary to talk explicitly about the weak nature of the neutrino energy-momentum measurement. The problem is that, as we are going to see in the next section, neutrino oscillation measurements are, in general, made in pre- and postselected ensembles, and the results of these measurements are not expectation values, but weak values, a concept introduced in 1988 by Aharanov, Albert and Vaidman (AAV) [31,43-45].

To understand the concept of weak value, suppose that after the measurement described by the Hamiltonian in (21) in the system ensemble $\Omega_{i}$, we focus only on the measurement outcomes from the system subensemble that ended up in some specific state $\left|\psi_{f}\right\rangle$. We name this subensemble $\Omega_{i f}$. We can find the statistics of the apparatus pointer variable by taking (24) and applying to it the system conditional final state

$$
\begin{aligned}
\left|\Omega_{i f}\right\rangle & =\left\langle\psi_{f} \mid \Omega_{f}\right\rangle=\left\langle\psi_{f} e^{-i \int \mathbf{H}_{i n t} d t} \mid \Omega_{i}\right\rangle \\
& =\left\langle\psi_{f} e^{i \mathbf{A x}_{D}} \mid \psi_{i}\right\rangle|\phi\rangle .
\end{aligned}
$$




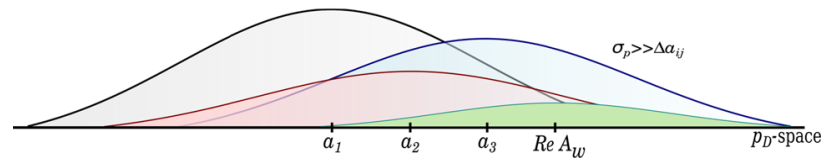

Fig. 4 Illustration of the resulting pointer wavefunction (green), with mean given by the real part of the weak value, $\operatorname{Re} A_{w}$, when, together with weak measurements, there is postselection of the state $\left|\psi_{f}\right\rangle$. Its amplitude is proportional to $\left\langle\psi_{f} \mid \psi_{i}\right\rangle$. On purpose, $\operatorname{Re} A_{w}$ is shown outside the range of eigenvalues $a_{i}$, to highlight one of the most interesting properties of weak values

Now, we expand the exponential inside (33), to obtain

$\left|\Omega_{i f}\right\rangle \approx\left\langle\psi_{f} \mid \psi_{i}\right\rangle\left(1+i A_{w} \mathbf{x}_{D}-\frac{1}{2} A_{w}^{2} \mathbf{x}_{D}^{2}+\cdots\right)|\phi\rangle$,

where $A_{w}^{n}$ is called the nth order weak value of $\mathbf{A}, A_{w}^{n} \equiv$ $\frac{\left\langle\psi_{f} \mathbf{A}^{n} \mid \psi_{i}\right\rangle}{\left\langle\psi_{f} \mid \psi_{i}\right\rangle}$. We use the hypothesis of weak measurements to argue that, for the apparatus, the action of the Hamiltonian in (21) is just a small perturbation and we truncate the expansion at first order in $\mathbf{x}_{D}$,

$\left\langle p_{D} \mid \Omega_{i f}\right\rangle \approx\left\langle\psi_{f} \mid \psi_{i}\right\rangle\left\langle p_{D} 1+i A_{w} \mathbf{x}_{D} \mid \phi\right\rangle$,

using the approximation $1+i A_{w} \mathbf{x}_{D} \approx e^{i A_{w} \mathbf{x}_{D}}$, which acts as a translation operator in $p_{D}$-space,

$\left\langle p_{D} 1+i A_{w} \mathbf{x}_{D} \mid \phi\right\rangle \approx\left\langle p_{D}-A_{w} \mid \phi\right\rangle$.

Assuming for the state of the pointer before the measurement,

$\left\langle p_{D} \mid \phi\right\rangle=\phi\left(p_{D}\right) \propto e^{-\frac{p_{D}^{2}}{4 \sigma_{p}^{2}}}$, with $\sigma_{x} \sigma_{p}=\frac{1}{2}$,

and that $A_{w}$ is a complex number, $A_{w}=\operatorname{Re} A_{w}+i \operatorname{Im} A_{w}$, then, the probability distribution for the apparatus after the measurement is

$$
\begin{aligned}
\left|\left\langle p_{D}-\operatorname{Re} A_{w}-i \operatorname{Im} A_{w} \mid \phi\right\rangle\right|^{2} & \propto e^{\frac{\left(\operatorname{Im} A_{w}\right)^{2}}{2 \sigma_{p}^{2}}} e^{\left.-\frac{\left(p_{D}-\operatorname{Re} A\right.}{2 \sigma_{p}^{2}}\right)^{2}} \\
& \approx\left|\phi\left(p_{D}-\operatorname{Re} A_{w}\right)\right|^{2}
\end{aligned}
$$

which is proportional to the initial probability distribution translated by $\operatorname{Re} A_{w}$ in $p_{D}$-space. ${ }^{10}$ Therefore, instead of moving the pointer to some eigenvalue, as in (26), a weak measurement with pre- and postselection move the pointer to the real part of the observable weak value, see Fig. 4.

\footnotetext{
10 Notice that we neglected the factor $e^{\frac{(\mathrm{Im} A w)^{2}}{2 \sigma_{p D}^{2}}}$ after ' $\approx$ ' sign in (38). This term has impact on the normalization of the pointer wavefunction after the measurement but does not change the center of the final distribution. Physically, it is related to the "back-action" on the variable $x_{D}$ due to the sudden change in $p_{D}$. In weak limit, of very little disturbance, the correction coming from such term is assumed to be negligible [43].
}

We usually think in eigenvalues as the only possible answers to single quantum measurements, but this section teaches us that with enough uncertainty in the detection and postselection, an entirely new type of answer appears: the weak value, $A_{w}$. The weak value is considered a property of a single system under pre- and postselection, revealed by a single measurement [46]. All physical consequences of the interactions of the system under such circumstances depend on $A_{w}$. Weak values can lie beyond the range of eigenvalues of A, so-called anomalous weak values [47,48], as illustrated in Fig. 4.

Observe in Fig. 4 that, due to the uncertainty in detection process, the eigenfunctions of observable A look like wave-packets with mean values $a_{i}$. Under general pre- and postselection, i.e. initial and final states are not restricted to be eigenvectors of the observable $\mathbf{A}$ - an effective wavepacket emerges with the mean value given by $\operatorname{Re} A_{w}$. Something analogous will happen to neutrinos in the next section, where weak values of energy and momentum will characterize them.

\section{Neutrino oscillations and the weak regime}

In this section we adapt the weak values formalism to neutrino detection. Start by interpreting the detection region (see discussion before (15)), as an apparatus (or pointer) that will measure the neutrino energy and momentum with uncertainties $\sigma_{e}$ and $\sigma_{p}$, respectively. In the relativistic onedimensional case, it is redundant to talk in terms of momentum and energy; then, in the following, we refer to momentum measurement. In the pointer interpretation, (20) must be understood in the same sense as (29):

$\sigma_{p}>>\Delta p_{a b}$. (Weak measurement)

As an apparatus, the detection region has conjugate variables $\mathbf{p}_{D}$ and $\mathbf{x}_{D}$ obeying $\left[\mathbf{x}_{D}, \mathbf{p}_{D}\right]=i$. In $p_{D}$-space, its wavefunction, $\phi\left(p_{D}\right)=\left\langle p_{D} \mid \phi\right\rangle$, is given by the combination of the production and detection Gaussian envelopes in (2) and (9), respectively: ${ }^{11}$

$\phi\left(p_{D}\right)=\phi^{P}\left(p_{D}\right) \phi^{D}\left(p_{D}\right) \propto e^{-\frac{p_{D}^{2}}{4 \sigma_{p P}^{2}}} e^{-\frac{p_{D}^{2}}{4 \sigma_{p D}^{2}}}=e^{-\frac{p_{D}^{2}}{4 \sigma_{P}^{2}}}$,

where we used (15). Thus, we model the detection region as a Gaussian pointer with resolution $\sigma_{p}$, as in the previous section. Here, $\sigma_{p}$ is the momentum resolution in neutrino detection, according to (15).

\footnotetext{
11 Actually, it is just after the measurement, $p_{D} \rightarrow p_{D}-p_{a}$, that $\phi_{P}$ and $\phi_{D}$ will be equal to the Gaussian envelopes in (2) and (9).
} 
We construct the Hamiltonian coupling the neutrino momentum, $\mathbf{p}$, to the pointer conjugate variable, $\mathbf{x}_{D}$ as ${ }^{12}$

$\mathbf{H}_{i n t}(t)=-\delta(t-T) \mathbf{p} \mathbf{x}_{D}$,

where $T$ is the average time of detection. According to this Hamiltonian, in analogy with (23), after measurement (assuming the initial value of $p_{D}$ is zero):

$\mathbf{p}_{D}(t>T)=\mathbf{p}(t=T)$.

In case the measurement is made for a neutrino mass eigenstate $v_{a}$, described by (1), we have, in Heisenberg picture (initial state $\left|v_{a}^{P}\right\rangle|\phi\rangle$ ),

$$
\begin{aligned}
\left\langle v_{a}^{P}\left\langle\phi \mathbf{p}_{D}(t>T) \mid \phi\right\rangle \mid v_{a}^{P}\right\rangle & =\langle\phi \mid \phi\rangle\left\langle v_{a}^{P} \mathbf{p}(t=T) \mid v_{a}^{P}\right\rangle \\
& =p_{a},
\end{aligned}
$$

with $|\phi\rangle$ the (normalized) state of the detection region. In other words, the detection region momentum distribution after the measurement $(t>T)$ is clustered around $p_{D}=p_{a}$, given in (6), as expected. This is just telling us that the detection process behaves as if a wave-packet with mean momentum $p_{a}$ and uncertainty $\sigma_{p}$ just arrived. In our example, if $p_{a}$ is known, the corresponding energy, $\epsilon_{a}$, is also known.

The next subsection is devoted to the most general case of coherent detection of several mass eigenstates with postselection (detection of a specific flavor). Weak values naturally appear.

\subsection{Neutrino oscillations with pre- and postselection}

In case the neutrino is preselected in the state $\left|v_{\alpha}^{P}\right\rangle$, evolves freely to $\left|v_{\alpha}^{P}(L, T)\right\rangle$, until being detected and, consequently, postselected in the state $\left|v_{\beta}^{D}\right\rangle$, we can write for the initial state

$\left|\Omega_{\alpha}\right\rangle=\left|v_{\alpha}^{P}(L, T)\right\rangle|\phi\rangle$.

In analogy with (33),

$$
\begin{aligned}
\left|\Omega_{\alpha \beta}(L, T)\right\rangle & =\left\langle v_{\beta}^{D} \mid \Omega_{\alpha}\right\rangle \\
& =\left\langle v_{\beta}^{D} e^{-i \int \mathbf{H}_{\text {int }} d t} \mid v_{\alpha}^{P}(L, T)\right\rangle|\phi\rangle \\
& =\left\langle v_{\beta}^{D} e^{i \mathbf{p x}_{D}} \mid v_{\alpha}^{P}(L, T)\right\rangle|\phi\rangle .
\end{aligned}
$$

Using the weak measurement hypothesis (39),

$$
\begin{aligned}
\left|\Omega_{\alpha \beta}(L, T)\right\rangle & \approx\left\langle v_{\beta}^{D} \mid v_{\alpha}^{P}(L, T)\right\rangle\left(1+i p_{w}^{\alpha \beta} \mathbf{x}_{D}\right)|\phi\rangle \\
& \approx\left\langle v_{\beta}^{D} \mid v_{\alpha}^{P}(L, T)\right\rangle\left|\phi\left(p_{D}-\operatorname{Re}\left\{p_{w}^{\alpha \beta}\right\}\right)\right\rangle,
\end{aligned}
$$

12 Notice that we are not imposing any kind of new interaction in the detection process, $\mathbf{H}_{i n t}$, here, is just an artifact of calculation.

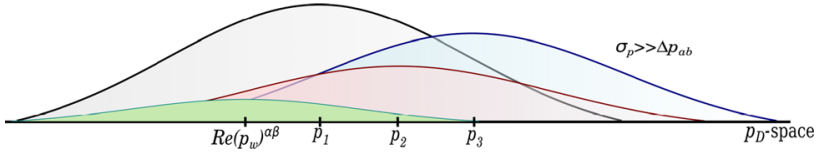

Fig. 5 Illustration of the weak measurement features of neutrino oscillations. The detection of the flavor $\left|v_{\beta}\right\rangle$ constitutes postselection. As usual, the resulting wave-packet (green) has mean given by $\operatorname{Re} p_{w}^{\alpha \beta}$ and we interpret it as the wave-packet of the detected $v_{\beta}$ or, generically, the "flavor wave-packet". Its amplitude is proportional to $\left\langle v_{\beta} \mid v_{\alpha}(L, T)\right\rangle$

where $p_{w}^{\alpha \beta}$ is also a function of $L$ and $T$, given by

$p_{w}^{\alpha \beta}(L, T)=\frac{\left\langle v_{\beta}^{D} \mathbf{p} \mid v_{\alpha}^{P}(L, T)\right\rangle}{\left\langle v_{\beta}^{D} \mid v_{\alpha}^{P}(L, T)\right\rangle}$.

Hence, the neutrino momentum measured by the particles in the detection region at average coordinates $(T, L)$ is given by $p_{D}=\operatorname{Re}\left\{p_{w}^{\alpha \beta}(L, T)\right\}$. Analogously, the energy is the real part of

$\epsilon_{w}^{\alpha \beta}(L, T)=\frac{\left\langle v_{\beta}^{D} \mathbf{H} \mid v_{\alpha}^{P}(L, T)\right\rangle}{\left\langle v_{\beta}^{D} \mid v_{\alpha}^{P}(L, T)\right\rangle}$

Notice that the flavor neutrino behaves as a single particle wave-packet with average energy and momentum $\operatorname{Re} \epsilon_{w}^{\alpha \beta}$ and $\operatorname{Re} p_{w}^{\alpha \beta}$ at $(T, L)$, see Fig. 5, in the same sense the massive wave-packets have averages $\epsilon_{a}$ and $p_{a}$.

\subsection{Normalization and probability current}

In this subsection, we work with one massive neutrino $v_{a}$, mass $m_{a}$, and explain how to write its wavefunction, probability density, and current satisfying the pointer interpretation. This is a standard treatment for massive particles, such as electrons or muons. In the next subsection, we mix the massive neutrinos and find an analogous treatment for flavor neutrinos.

Equations (27) and (40) imply that for our interpretation of the detection region as a pointer, the production and detection Gaussian envelopes should not be normalized separately but in a correlated manner

$$
\int d p\left|\phi\left(p-p_{a}\right)\right|^{2}=\int d p\left|\phi^{P}\left(p-p_{a}\right)\right|^{2}\left|\phi^{D}\left(p-p_{a}\right)\right|^{2}=1,
$$

and therefore

$\phi\left(p-p_{a}\right)=\frac{1}{\left(2 \pi \sigma_{p}^{2}\right)^{\frac{1}{4}}} e^{\frac{\left(p-p_{a}\right)^{2}}{4 \sigma_{p}^{2}}}$. 
The coordinate space wavefunction at time $T$ and position $L$ for this particle is, in analogy with (3), the space-time integral of $\phi\left(p-p_{a}\right)$ :

$$
\begin{aligned}
A_{a}(L, T) & =\left\langle v_{a}^{D} \mid v_{a}^{P}(L, T)\right\rangle \\
& =\int \frac{d p}{\sqrt{2 \pi} \sqrt{E_{a}(p)}} \phi\left(p-p_{a}\right) e^{-i E_{a}(p) T+i p L} .
\end{aligned}
$$

For sharply peaked wave-packets,

$$
\begin{aligned}
& A_{a}(L, T) \approx \int \frac{d p}{\sqrt{2 \pi} \sqrt{2 \epsilon_{a}}} \phi\left(p-p_{a}\right) e^{-i \epsilon_{a} T} e^{-i v_{a}\left(p-p_{a}\right) T} e^{i p L} \\
&=\frac{1}{\sqrt{2 \pi} \sqrt{2 \epsilon_{a}}}\left(\frac{2 \pi}{\sigma_{x}^{2}}\right)^{\frac{1}{4}} \\
& \times \exp \left(-i \epsilon_{a} T+i p_{a} L-\frac{\left(L-v_{a} T\right)^{2}}{4 \sigma_{x}^{2}}\right) .
\end{aligned}
$$

Relativistic particles with defined masses, such as $v_{a}$, obey Klein-Gordon equation. ${ }^{13}$ From (52), the Klein-Gordon current for an arbitrary particle produced as $\left|\nu_{a}^{P}\right\rangle$ and detected as $\left|v_{a}^{D}\right\rangle$ after propagating a distance $L$ during some time $T$ is given by

$$
J_{a}(L, T)=2 p_{a}\left|A_{a}(L, T)\right|^{2} .
$$

Together with the probability density, $\rho_{a}(L, T)=2 \epsilon_{a} \mid A_{a}(L, T)$ $1^{2}, J_{a}(L, T)$ satisfies the Klein-Gordon continuity equation,

$$
\frac{\partial}{\partial T} \rho_{a}(L, T)+\frac{\partial}{\partial L} J_{a}(L, T)=0 .
$$

What we call probability density is actually a number (of particles) density. The way $A_{a}(L, T)$ is normalized in (51), however, is so that there is only one massive neutrino $v_{a}$ in the whole space at any given time:

$$
\int d L \rho_{a}(L, T)=1 \text {. }
$$

Note that, with such a convention, $\rho_{a}$ has dimension of $1 /$ length as it should be in a one-particle theory and $J_{a} \propto$ $1 /$ time. Therefore, integrating $J_{a}$ for the whole time of the experiment should give us the probability that, after the detection, the detector will register a particle of index $a$ :

$P_{a}(L)=\int d T J_{a}(L, T)=1$,

as expected.

\footnotetext{
$\overline{13}$ As matter of fact, $v_{a}$ obeys Dirac equation. Klein-Gordon density and currents are approximations of their respective Dirac counterparts when spinor degrees of freedom are ignored. The calculations in the following can be reproduced without ignoring the spinors by using Gordon decomposition [50].
}

In the next section, we illustrate the role of weak values in neutrino oscillations by finding the appropriate currents for flavor neutrinos.

\subsection{Neutrino oscillation probability}

In analogy with Eq. (53) and $\operatorname{Re}\left\{p_{w}^{\alpha \beta}\right\}$ in place of $p_{a}$ :

$$
J_{\alpha \beta}(L, T)=2 \operatorname{Re}\left\{p_{w}^{\alpha \beta}\right\}\left|A_{\alpha \beta}(L, T)\right|^{2},
$$

with $A_{\alpha \beta}(L, T)=\sum_{a} U_{\alpha a}^{*} U_{\beta a} A_{a}(L, T)$. At order $\frac{m^{2}}{E^{2}}, J_{\alpha \beta}$ obeys a continuity equation of the form:

$$
\sum_{\beta}\left(\frac{\partial}{\partial T} \rho_{\alpha \beta}(L, T)+\frac{\partial}{\partial L} J_{\alpha \beta}(L, T)\right)=0,
$$

with $\rho_{\alpha \beta}(L, T)=2 \operatorname{Re}\left\{\epsilon_{w}^{\alpha \beta}\right\}\left|A_{\alpha \beta}(L, T)\right|^{2}$. Indeed, (57) and (58) can be derived from manipulating Klein-Gordon equation without ever referring to weak measurements. On the other hand, weak measurements provide a physical interpretation. Different from (53), (57) describes a probability that is not conserved, in general, due to flavor transformations.

The time-independent flavor oscillation probability is defined as the probability current integrated over the area of the detector for the entire time duration of the experiment,

$P_{\alpha \beta}(\vec{L})=\int d T \int_{S} d A J_{\alpha \beta}(\vec{L}, T)$

Since we are working in just one dimension, this integral simplifies to

$P_{\alpha \beta}(L)=\int d T \quad J_{\alpha \beta}(L, T)$.

This is equivalent to (56) in the context of mixed particles, it gives the probability that the detector will register an index $\beta$. Substituting (57) into (60), we have

$$
\begin{aligned}
P_{\alpha \beta}(L)= & \int d T 2 \operatorname{Re}\left\{p_{w}^{\alpha \beta}\right\}\left|A_{\alpha \beta}(L, T)\right|^{2} \\
= & 2 \operatorname{Re} \sum_{a, b} U_{\alpha a}^{*} U_{\beta a} U_{\alpha b} U_{\beta b}^{*} \\
& \times \int d T\left\langle v_{b}^{P}(L, T) \mid v_{b}^{D}\right\rangle\left\langle v_{a}^{D} \mathbf{p} \mid v_{a}^{P}(L, T)\right\rangle .
\end{aligned}
$$

Now, $\left\langle v_{a}^{D} \mid v_{a}^{P}(L, T)\right\rangle \approx A_{a}(L, T)$ as given by (52). For the second term in (61), and

$$
\left\langle v_{a}^{D} \mathbf{p} \mid v_{a}^{P}(L, T)\right\rangle \approx\left(p_{a}+2 i \frac{L-v_{a} T}{4 \sigma_{x}^{2}}\right) A_{a}(L, T)
$$


Back to (61), and using relations (4) and (6), we integrate ${ }^{14}$

$$
\begin{aligned}
P_{\alpha \beta}(L) \approx & \operatorname{Re}\left\{\sum_{a, b} U_{\alpha a}^{*} U_{\beta a} U_{\alpha b} U_{\beta b}^{*} \sqrt{\frac{2}{v_{a}^{2}+v_{b}^{2}}} p_{a} \frac{1}{\sqrt{\epsilon_{a}}}\right. \\
& \left.\times \frac{1}{\sqrt{\epsilon_{b}}} e^{-i \frac{\Delta m_{a b}^{2} L}{2 E}} e^{-\left(\frac{L}{L_{c o h}^{a b}}\right)^{2}} e^{-\frac{\left(\Delta \epsilon_{a b}\right)^{2}}{8 \sigma_{e}^{2}}}\right\} .
\end{aligned}
$$

$P_{\alpha \beta}$ is dimensionless and normalized (recall (19) and related discussion). When the dependence of $p_{a}, \epsilon_{a}$ and $v_{a}$ on the index $a$ is negligible,

$$
\begin{aligned}
P_{\alpha \beta}(L) \approx & \sum_{a, b} U_{\alpha a}^{*} U_{\beta a} U_{\alpha b} U_{\beta b}^{*} \\
& \times e^{-i \frac{\Delta m_{a b}^{2} L}{2 E}} e^{-\left(\frac{L}{L_{c o h}^{a b}}\right)^{2}} e^{-\frac{\left(\Delta \epsilon_{a b}\right)^{2}}{8 \sigma_{e}^{2}}},
\end{aligned}
$$

and we reach the standard oscillation probability.

Of course, by neglecting the impact of the mass eigenstate indexes in $p_{a}, \epsilon_{a}$ and $v_{a}$, we are neglecting the impact of the weak values on the phenomenological formula (64) itself. ${ }^{15}$ But the point here is that to find Eq. (57) was regarded as a conceptual problem in some previous references (check [34-37]). The argument is that Hilbert spaces and probability currents are unequivocally defined for particles with definite mass, which is not the case of neutrinos. However, such problems do not arise in postselected quantum mechanics, where the intermediate process is not an ordinary quantum state, described by one ket, but a pre- and postselected state, characterized by two kets at the same space-time point and the weak value.

\section{Conclusion}

This paper gives an overview of both the theory of neutrino oscillations and the basic von Neumann measurement theory. An advantage of the von Neumann model is that we can embed on it quantum mechanical uncertainties and model the coherent detection of neutrino wave-packets by treating the measuring apparatus as a quantum system. When these uncertainties are sufficiently large, see (29), we call it a weak measurement. We describe neutrino oscillations as a postselected weak measurement with the detected wavefunction mean value given by the weak value; see Eq. (47) and Fig. 5. It is then straightforward to write down the currents and densities for the postselected wavefunctions and reach the probability's usual formula.

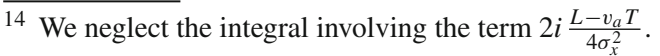

15 Although in principle one could test the theory presented here by detecting anomalous weak values [31], anything of order $\frac{m^{2}}{E}$ impacting energy or momentum measurement is impractical.
}

Acknowledgements This study was financed in part by the Coordenação de Aperfeiçoamento de Pessoal de Nível Superior - Brasil (CAPES) - Finance Code 001. YPPS acknowledges support from FAPESP funding Grants No. 2014/19164- 453 6, No. 2017/05515-0 and No. 2019/22961-9. MCO also acknowledges support from CNPq. YPPS is thankful to O. L. G. Peres, M. E. Chaves, A. Y. Smirnov and E. Akhmedov for enlightening discussions on wave-packets and neutrino oscillations.

Data Availability Statement This manuscript has no associated data or the data will not be deposited. [Authors' comment: This is a theoretical study and no experimental data.]

Open Access This article is licensed under a Creative Commons Attribution 4.0 International License, which permits use, sharing, adaptation, distribution and reproduction in any medium or format, as long as you give appropriate credit to the original author(s) and the source, provide a link to the Creative Commons licence, and indicate if changes were made. The images or other third party material in this article are included in the article's Creative Commons licence, unless indicated otherwise in a credit line to the material. If material is not included in the article's Creative Commons licence and your intended use is not permitted by statutory regulation or exceeds the permitted use, you will need to obtain permission directly from the copyright holder. To view a copy of this licence, visit http://creativecomm ons.org/licenses/by/4.0/.

Funded by $\mathrm{SCOAP}^{3}$.

\section{References}

1. Y. Fukuda et al. (Super-Kamiokande), Phys. Rev. Lett. 81, 1562 (1998). arXiv:hep-ex/9807003

2. Q.R. Ahmad et al. (SNO), Phys. Rev. Lett. 89, 011301 (2002). arXiv:nucl-ex/0204008

3. T. Araki et al. (KamLAND), Phys. Rev. Lett. 94, 081801 (2005). arXiv:hep-ex/0406035

4. B. Pontecorvo, Sov. Phys. JETP 6, 429 (1957). [Zh. Eksp. Teor. Fiz. 33, 549 (1957)]

5. Z. Maki, M. Nakagawa, S. Sakata, Prog. Theor. Phys. 28, 870 (1962)

6. B. Pontecorvo, Sov. Phys. JETP 26, 984 (1968)

7. B. Kayser, Phys. Rev. D 24, 110 (1981)

8. R.G. WinterLett, Nuovo Cim. 30, 101 (1981)

9. C. Giunti, C.W. Kim, Found. Phys. Lett. 14, 213 (2001). arXiv:hep-ph/0011074

10. S. Nussinov, Phys. Lett. 63B, 201 (1976)

11. C. GiuntiC, W. KimU, W. Lee, Phys. Rev. D 44, 3635 (1991)

12. J. Rich, Phys. Rev. D 48, 4318 (1993)

13. C. GiuntiC, W. Kim, Phys. Rev. D 58, 017301 (1998). arXiv:hep-ph/9711363

14. K. Kiers, S. Nussinov, N. Weiss, Phys. Rev. D 53, 537 (1996). arXiv:hep-ph/9506271 [hep-ph]

15. K. Kiers, N. Weiss, Phys. Rev. D 57, 3091 (1998). arXiv:hep-ph/9710289 [hep-ph]

16. E.K. Akhmedov, A.Yu. Smirnov, Phys. Atom. Nucl. 72, 1363 (2009). arXiv:0905.1903 [hep-ph]

17. E. Akhmedov, JHEP 07 070. arXiv:1703.08169 [hep-ph]

18. E. Akhmedov, Quantum mechanics aspects and subtleties of neutrino oscillations (2019). arXiv:1901.05232 [hep-ph]

19. I.Y. Kobzarev, B.V. Martemyanov, L.B. OkunM, G. Shchepkin, Sov. J. Nucl. Phys. 35, 708 (1982)

20. C. Giunti, C.W. Kim, J.A. Lee, U.W. Lee, Phys. Rev. D 48, 4310 (1993). arXiv:hep-ph/9305276 
21. M. Blasone, G. Vitiello, Ann. Phys. 244, 283 (1995). [Erratum: Ann. Phys. 249, 363 (1996)]. arXiv:hep-ph/9501263

22. W. Grimus, P. Stockinger, Phys. Rev. D 54, 3414 (1996). arXiv:hep-ph/9603430

23. M. Beuthe, Phys. Rep. 375, 105 (2003). arXiv:hep-ph/0109119

24. M. Beuthe, Phys. Rev. D 66, 013003 (2002). arXiv:hep-ph/0202068

25. C. Giunti, JHEP 11 017. arXiv:hep-ph/0205014

26. E.K. Akhmedov, J. Kopp, JHEP 04, 008. [Erratum: JHEP 10, 052 (2013)]. arXiv:1001.4815 [hep-ph]

27. A. Kobach, A.V. Manohar, J. McGreevy, Phys. Lett. B 783, 59 (2018). arXiv:1711.07491

28. E. Arthurs, J.L. Kelly, Bell Syst. Tech. J. 44, 725 (1965)

29. S. Gudder, Fuzzy Sets Syst. 155, 18 (2005) (measures and conditioning)

30. C.A. Fuchs, A. Peres, Phys. Rev. 53, 2038 (1996). arXiv:quant-ph/9512023

31. Y. Aharonov, D.Z. Albert, L. Vaidman, Phys. Rev. Lett. 60, 1351 (1988)

32. Y. Aharonov, L. Vaidman, Phys. Rev. 41, 11 (1990)

33. J.M. Johansen, Phys. Lett. A 322, 298-300 (2004)

34. M. Zralek, Acta Phys. Polon. B 29, 3925 (1998). arXiv:hep-ph/9810543

35. B. Ancochea, A. Bramon, R. Munoz-Tapia, M. Nowakowski, Phys. Lett. B 389, 149 (1996). arXiv:hep-ph/9605454

36. M. Blasone, P. Jizba, G. Vitiello, Phys. Lett. B 517, 471 (2001). arXiv:hep-th/0103087

37. M. Blasone, P. Pires Pacheco, H. Wan Chan Tseung, Phys. Rev. D 67, 073011 (2003). arXiv:hep-ph/0212402

38. S. Lloyd, J. Preskill, JHEP 08 126. arXiv:1308.4209 [hep-th]
39. C. Giunti, C.W. Kim, Fundamentals of Neutrino Physics and Astrophysics (Oxford University Press, Oxford, 2007)

40. J. Von Neumann, N.A. Wheeler, Mathematical foundations of quantum mechanics, New ed. (Princeton University Press, Princeton, 2018)

41. Y. Aharonov, D. Rohrlich, in Quantum Paradoxes: Quantum Theory for the Perplexed, ed. by Y. Aharonov, D. Rohrlich. (WileyVCH, 2003), p. 299 (ISBN 3-527-40391-4)

42. Y. Aharonov, A. Botero, Phys. Rev. A 72 (2005). https://doi.org/ 10.1103/physreva.72.052111

43. J. Dressel, M. Malik, F.M. Miatto, A.N. Jordan, R.W. Boyd, Rev. Mod. Phys. 86, 307-316 (2014)

44. Y. Shikano, Theory of "weak value" and quantum mechanical measurements (2011). arXiv:1110.5055 [quant-ph]

45. L. Qin, W. Feng, X.-Q. Li, Sci. Rep. 6 (2016). https://doi.org/10. 1038/srep20286

46. L. Vaidman, A. Ben-Israel, J. Dziewior, L. Knips, M. Weiß1, J. Meinecke, C. Schwemmer, R. Berand, H. Weinfurter, Phys. Rev. A 96 (2017). https://doi.org/10.1103/physreva.96.032114

47. Y. Aharonov, F. Colombo, I. Sabadini, D.C. Struppa, J. Tollaksen, J. Phys. A Math. Theor. 44, 365304 (2011)

48. M.V. Berry, P. Shukla, J. Phys. A Math. Theor. 45, 015301 (2011)

49. M.V. Berry, N. Brunner, S. Popescu, P. Shukla, J. Phys. A 44, 492001 (2011). arXiv:1110.2832 [hep-ph]

50. J. Sakurai, Advanced Quantum Mechanics, Addison-Wesley Series in Advanced Physics (Addison-Wesley, 1987) 